Review

\title{
Extrapolation of Transport Properties and Figure of Merit of a Thermoelectric Material
}

\section{H. Julian Goldsmid ${ }^{1, *}$ and Jeff Sharp ${ }^{2}$}

1 School of Physics, University of New South Wales, Sydney 2052, Australia

2 Marlow Industries, Inc. (Subsidiary of II-VI Incorporated) 10451 Vista Park Rd., Dallas, TX 75238, USA; E-Mail: jsharp@marlow.com

* Author to whom correspondence should be addressed; E-Mail: hjgoldsmid@bigpond.com; Tel.: +61-3-62291776.

Academic Editor: Sima Aminorroaya-Yamini

Received: 11 May 2015 / Accepted: 15 June 2015 / Published: 26 June 2015

\begin{abstract}
The accurate determination of the thermoelectric properties of a material becomes increasingly difficult as the temperature rises. However, it is the properties at elevated temperatures that are important if thermoelectric generator efficiency is to be improved. It is shown that the dimensionless figure of merit, $Z T$, might be expected to rise with temperature for a given material provided that minority carrier conduction can be avoided. It is, of course, also necessary that the material should remain stable over the whole operating range. We show that the prediction of high temperature properties in the extrinsic region is possible if the temperature dependence of carrier mobility and lattice thermal conductivity are known. Also, we show how the undesirable effects arising from mixed or intrinsic conduction can be calculated from the energy gap and the relative mobilities of the electrons and the positive holes. The processes involved are discussed in general terms and are illustrated for different systems. These comprise the bismuth telluride alloys, silicon-germanium alloys, magnesium-silicon-tin and higher manganese silicide.
\end{abstract}

Keywords: thermoelectric; generation; semiconductors; energy conversion; energy gap; intrinsic conduction; extrinsic conduction 


\section{Introduction}

There is increasing interest in the use of thermoelectric generation to convert heat into electrical energy. As for other types of heat engine, the efficiency of a thermoelectric generator depends on the temperature difference between the source and the sink, so it is desirable that the source of heat be at as high a temperature as possible. It is necessary, therefore, that the Seebeck coefficient, $\alpha$, the electrical conductivity, $\sigma$, and the thermal conductivity, $\lambda$, be known at high temperatures. These quantities are combined in what is known as the figure of merit [1], $Z$, which is defined as $\alpha^{2} \sigma / \lambda$, usually appearing in its dimensionless form, $Z T$.

In actual fact, the figure of merit should really be defined for a pair of thermoelements having Seebeck coefficients of opposite sign [2]. Thus,

$$
Z=\frac{\left(\alpha_{p}-\alpha_{n}\right)^{2}}{\left\{\left(\lambda_{p} \rho_{p}\right)^{1 / 2}+\left(\lambda_{n} \rho_{n}\right)^{1 / 2}\right\}^{2}}
$$

where the subscripts $p$ and $n$ denote the two types of material. The maximum efficiency, $\phi$, of the couple is given by:

$$
\phi=\frac{\left(T_{1}-T_{2}\right)(M-1)}{T_{1}\left(M+T_{2} / T_{1}\right)}
$$

where $M=(1+Z T)^{1 / 2}$. It is supposed that the temperature difference, $\left(T_{1}-T_{2}\right)$, between the source and sink is rather small compared with the absolute temperature, or else some sort of averaging process must be used in expressing $M$.

The ratio $\left(T_{1}-T_{2}\right) / T_{1}$ is the efficiency of a Carnot cycle operating between these temperatures. It is clear that the efficiency also depends on the quantity $M$ and, thus, on $Z T$.

It is convenient for us to select the positive and negative materials separately, and it is usual for $Z T$ to be optimised for a single material, with the value of $Z T$ for a couple then being taken as the average for the two branches, usually a good approximation. The value of $Z T$ for a single material is indeed the relevant quantity in Equation (2) if the ratio of electrical to thermal conductivity is very large, as it is when the second branch is a superconductor $[3,4]$, but this does not become a consideration at the appropriate temperatures for thermoelectric generation.

Thermoelectric modules for refrigeration using the Peltier effect are widely available. They usually consist of positive and negative thermoelements made from alloys of bismuth telluride with antimony telluride and bismuth selenide [5]. The composition of the alloys is generally optimised so that the figure of merit, ZT, is highest near or below room temperature. These modules are often employed for thermoelectric generation when the temperature of the heat source is not too high. As the temperature is raised, alloys of similar composition may be used but the carrier concentrations should be modified by doping so that $Z T$ is optimised for a different region. It may also be necessary to change the construction of the module so that it can withstand the rise in temperature.

There is obviously a temperature above which the bismuth telluride alloys can no longer be used and other compounds and alloys are needed. These, in turn, have to be replaced when the temperature of the source is further increased. Clearly, then, there are several different systems of materials that must be investigated in designing thermoelectric generators, in contrast with the situation in thermoelectric 
refrigeration. Although the figure of merit remains the most important parameter, it also may be necessary to take account of the compatibility factor [6] when different materials are incorporated in the modules.

The accurate measurement of the thermoelectric properties of any material becomes more and more difficult as the temperature rises [7,8]. Very often the published data cover only a limited range. Thus, it is useful to be able to estimate the high temperature properties from observations at much lower temperatures.

It is evident that a thermoelectric material that is the best over a certain range will not necessarily be superior at some higher temperature. It may be subject to a phase change, chemical deterioration or, perhaps, melting. However, even if such events do not occur, the thermoelectric properties may themselves fall away. At the very least, one would expect to have to change the doping level to restore the Fermi energy to its optimum value. Also, unless the energy gap is large enough, one would expect deterioration in the figure of merit to result from the presence of minority carriers. To some extent, the effect of minority carriers can be reduced by increasing the concentration of dopant; the optimum Seebeck coefficient becomes lower when the energy gap is small enough for minority carrier conduction to be a factor.

We shall first show how the thermoelectric parameters will change with rise of temperature when there is only one type of carrier. We shall then determine the effects of minority carrier conduction when the energy gap is too small to prevent their appearance. Finally, we shall illustrate the principles that we have outlined for some typical systems, including the bismuth telluride alloys, silicon-germanium alloys, magnesium-silicon-tin and higher manganese silicides.

\section{Variation of the Thermoelectric Properties with Temperature for an Extrinsic Conductor}

Let us suppose that the transport properties for a given material have been determined over a specific temperature range, and that it is desired to predict the thermoelectric performance above this region. From the lower temperature properties one should be able to determine the quantity known as $\beta$ that was first defined by Chasmar and Stratton [9] as $(k / e)^{2} \sigma_{0} T / \lambda_{\mathrm{L}}$, where $\sigma_{0}$ is equal to $2 e \mu\left(2 \pi m * k T / h^{2}\right)^{3 / 2}$. Here $\mu$ is the mobility of the majority carriers, $\lambda_{\mathrm{L}}$ is the lattice thermal conductivity, and $m *$ is the density-of-states effective mass. The quantity, $\beta$, is not constant, since the carrier mobility and the lattice thermal conductivity depend on temperature. Thus, we need to predict the temperature-variation of both quantities. There may also be a change in the effective mass and the emergence of extra bands but, for the moment, we concentrate on $\mu$ and $\lambda_{\mathrm{L}}$.

It is helpful to discuss the figure of merit, $Z$, as the ratio of a quantity known as the power factor $\alpha^{2} \sigma$ to the thermal conductivity, $\lambda$. The power factor depends solely on the electronic properties of the material whereas the thermal conductivity includes, and may be dominated by, the lattice contribution.

The calculations are simplest when acoustic-mode lattice scattering of the carriers takes place, since the mobility is then proportional to $T^{-3 / 2}$ and $\sigma_{0}$ is independent of temperature, provided that the effective mass is constant. The electrical conductivity for a given value of the Seebeck coefficient should not change with temperature. The temperature dependence of $Z T$ is then controlled by the thermal conductivity, which is the sum of $\lambda_{\mathrm{L}}$ and the electronic component $\lambda_{\mathrm{e}}$. 
$\lambda_{\mathrm{e}}$ is equal to $L \sigma T$, where the Lorenz number, $L$, is given by $A(k / e)^{2}$, with the factor $A$ equal to $\pi^{2} / 3$ for a degenerate conductor. When acoustic mode lattice scattering is dominant, $A$ is equal to 2 in a non-degenerate conductor and is somewhat higher in a partially degenerate material.

For a pure crystalline material, the lattice conductivity varies as $T^{-1}$ and $\beta$ is proportional to $T^{2}$. On the other hand, when point defect scattering of the phonons is completely dominant, we might expect $\lambda_{\mathrm{L}}$ to be independent of temperature and $\beta$ to vary as $T$. Usually, the lattice thermal conductivity for a thermoelectric material will decrease as the temperature rises but not as rapidly as $1 / T$. For most semiconductors the lattice contribution to the thermal conductivity predominates over the electronic component but this is not necessarily true for thermoelectric materials. Nevertheless, it is not expected that the total thermal conductivity will rise with temperature, so $Z T$ should become greater as $T$ increases.

Once the variation with temperature of the carrier mobility and the lattice conductivity are known, it is a straightforward matter to calculate the thermoelectric properties at any temperature provided that minority carriers are absent. It must be borne in mind, though, that the optimum carrier concentration usually corresponds to a Fermi energy that is close to the edge of the conduction or valence band. In other words, the optimised material will almost certainly lie in the region of partial degeneracy.

The Seebeck coefficient, the electrical conductivity and the electronic thermal conductivity can all be expressed in terms of a set of integrals of the form

$$
K_{s}=\frac{8 \pi}{3}\left(\frac{2}{h^{2}}\right)^{3 / 2}\left(m^{*}\right)^{1 / 2} T \tau_{0}(s+r+3 / 2)(k T)^{s+r+3 / 2} F_{s+r+1 / 2}
$$

where

$$
F_{n}(\xi)=\int_{0}^{\infty} \xi^{n} f_{0}(\xi) d \xi
$$

Here the reduced energy, $\xi$, is equal to $E / k T$. The functions $F_{n}$ are the Fermi-Dirac integrals and have been tabulated $[1,10,11]$ for different values of the reduced Fermi energy, $\eta$, which is equal to $E_{F} / k T$.

When there is only one type of carrier, the expressions for the electrical conductivity, $\sigma$, the electronic thermal conductivity, $\lambda_{\text {e, }}$ and the Seebeck coefficient, $\alpha$, in terms of the integrals, $K_{\text {s, }}$ are:

$$
\begin{gathered}
\sigma=\frac{e^{2}}{T} K_{1} \\
\lambda_{e}=\frac{1}{T^{2}}\left(K_{2}-\frac{K_{1}^{2}}{K_{0}}\right)
\end{gathered}
$$

and

$$
\alpha= \pm \frac{1}{e T}\left(E_{F}-\frac{K_{1}}{K_{0}}\right)
$$

where the upper sign applies for electrons and the lower sign for holes. The total thermal conductivity $\lambda$ is equal to $\lambda_{\mathrm{e}}+\lambda_{\mathrm{L}}$, where $\lambda_{\mathrm{L}}$ is the lattice component. Thus, these equations allow us to determine the figure of merit. 


\section{Determination of the Thermoelectric Properties in the Mixed Conduction Regime}

We now turn to the calculation of the different parameters when the energy gap, $E_{\mathrm{g}}$, is small enough for minority carriers to be influential even for optimal $\eta$. We shall need to know both $E_{\mathrm{g}}$ and a quantity that we shall call $c$, the ratio of $\beta$ for the minority carriers to its value for the majority carriers.

It is obvious that minority carriers are undesirable since the electrons and holes have partial Seebeck coefficients of opposite sign. The magnitude of the overall Seebeck coefficient is then less than if only the majority carriers existed. Less obvious, but also important, is the bipolar contribution to the electronic thermal conductivity. When both electrons and holes are present, they can carry their activation energy (approximately equal to the energy gap), along the direction of the temperature gradient, without there being any net electric current. The bipolar thermal conductivity can be more than an order of magnitude greater than the electronic thermal conductivity calculated using the normal Lorenz number.

The energy gap may be estimated in a number of ways. For example, it can be found from the infra-red absorption edge or from the temperature dependence of the Hall coefficient. However, the most appropriate method for our purposes would seem to be that which requires the measurement of the maximum Seebeck coefficient as a function of temperature [12,13]. This method of estimating the energy gap has recently been discussed in detail by Gibbs et al. [14].

The maximum Seebeck coefficient depends primarily on the energy gap, but it also depends on the ratio, $\mathrm{c}$, of the $\beta$ values of the two carrier types, though it is to be hoped that $c$ may not need to be known accurately. Surprisingly, there seem to be few semiconductors for which $c$ is greatly different from unity [15]. For example, in $\mathrm{Bi}_{2} \mathrm{Te}_{3}, c$ is equal to 0.94 . However, there is a large difference in the $\beta$ values for the two types of carrier in Ge for which $c$ is equal to 0.18 (when the holes are the minority carriers). Although the energy gap is the major factor in determining the thermoelectric properties in the near intrinsic region, we cannot ignore the influence of $c$.

Expressions for the electrical and thermal conductivity and the Seebeck coefficient when there are two types of carrier can be found in review articles and textbooks on thermoelectricity [1].

When both types of carrier are present, the electrical conductivity is given by

$$
\sigma=\sigma_{1}+\sigma_{2}
$$

the Seebeck coefficient by

$$
\alpha=\frac{\alpha_{1} \sigma_{1}+\alpha_{2} \sigma_{2}}{\sigma_{1}+\sigma_{2}}
$$

and the electronic thermal conductivity by

$$
\lambda_{e}=\lambda_{\mathrm{e} 1}+\lambda_{e 2}+\frac{\sigma_{1} \sigma_{2}}{\sigma_{1}+\sigma_{2}}\left(\alpha_{1}-\alpha_{2}\right)^{2} T
$$

Equations (8)-(10) allow us to calculate the figure of merit $Z T$ in a mixed or intrinsic conductor.

It is noted that the various parameters are often expressed in dimensionless forms. For example, the Seebeck coefficients may be given in terms of $k / e$, which is equal to $86.2 \mu \mathrm{V} / \mathrm{K}$. Likewise, the dimensionless energy gap, $\eta_{\mathrm{g}}$, is equal to $E_{\mathrm{g}} / k T$. These dimensionless forms are used when convenient in the remainder of this article. 


\section{Estimation of the Band Gap and the Effect of the Parameter $c$}

We now discuss the determination of the band gap from the maximum Seebeck coefficient for a material such as germanium in which the parameter $c$ is not close to unity. We have calculated $2 \alpha_{\max } / \eta_{\mathrm{g}}$ for energy gaps from $5 k T$ to $30 k T$ and for $c$ from 0.2 up to 5 . The results are shown in Figure 1.

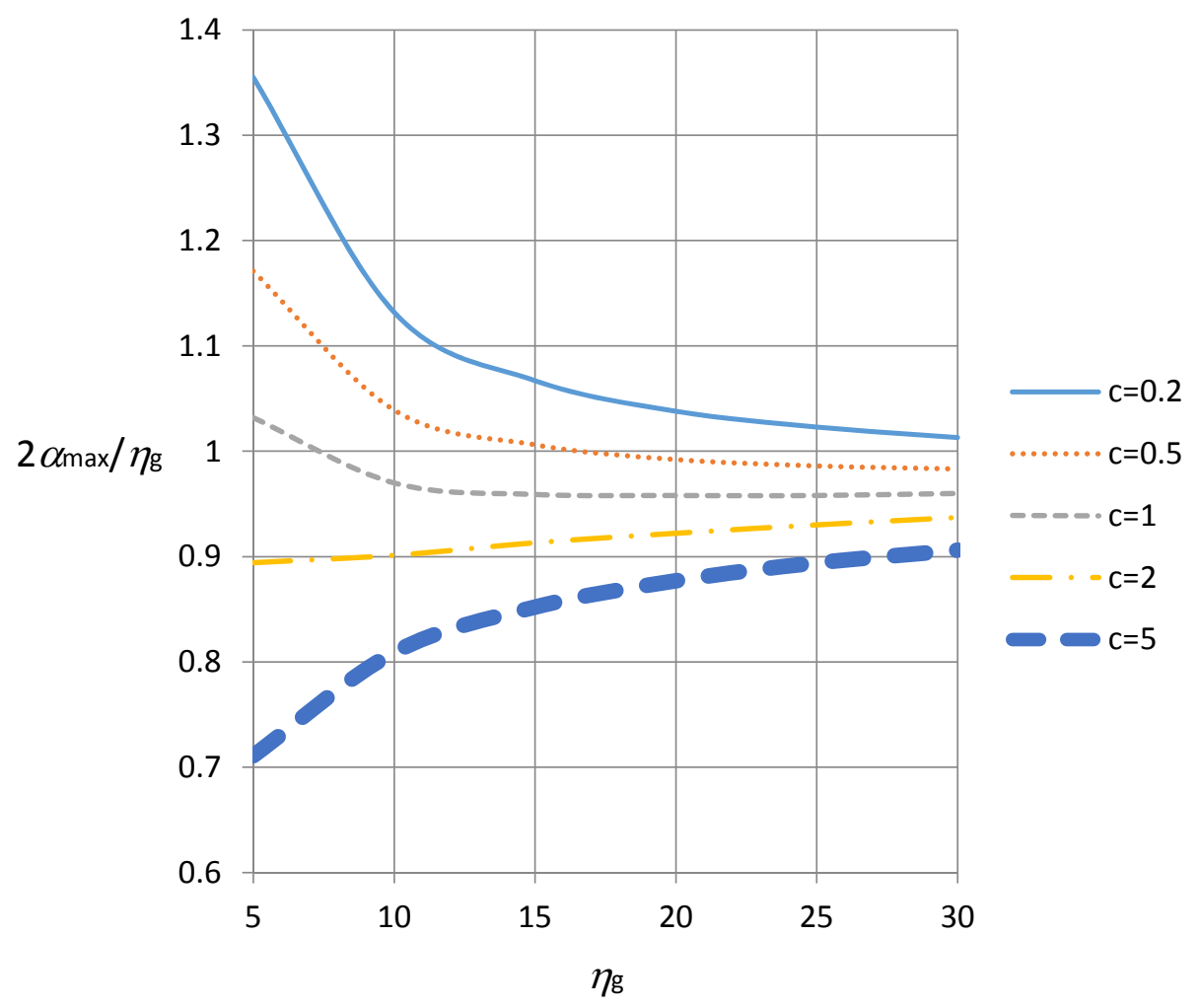

Figure 1. Plots of $2 \alpha_{\max } / \eta_{\mathrm{g}}$ against $\eta_{\mathrm{g}}$ for various values of the parameter c.

The so-called Goldsmid-Sharp method for determining the energy gap clearly becomes inaccurate when the energy gap is smaller than about $20 k T$. However, when $c=1,2 \alpha_{\max }$ and $\eta_{\mathrm{g}}$ are almost equal, the ratio $2 \alpha_{\max } / \eta_{\mathrm{g}}$ being about 0.96 for a wide range of $\eta_{\mathrm{g}}$. In other words, if $c \simeq 1$, we can generally suppose that the true dimensionless thermal gap is close to $2 \alpha_{\max } / 0.96$, which for practical purposes can be taken as $2 \alpha_{\text {max }}$.

We have not been able to identify any potential thermoelectric generator material with $c$ outside the limits of about 0.2 and 5, though, of course, semiconductors with such values of $c$ do exist. Near these extremes $2 \alpha_{\max } / \eta_{\mathrm{g}}$ varies quite considerably with the gap width. In the absence of knowledge of $c$ for a particular material, we might set $c=1$ and the apparent dimensionless energy gap equal to $2 \alpha$ max.

From the practical viewpoint it would be best to determine the maximum Seebeck coefficient for both n-type and p-type material. One could then get a reliable value of both the energy gap and $c$. Noting that $c=0.2$ for one type of carrier implies $c=5$ for the other type, we see that the geometric mean of the two values of $2 \alpha_{\mathrm{max}} / \eta_{\mathrm{g}}$ is almost equal to unity even when $\eta_{\mathrm{g}}$ is as small as 5 . 


\section{Thermoelectric Generation Using the Alloys of Bismuth Telluride}

Although the efficiency of conversion from heat to electricity generally improves as the temperature of the source rises, there are many applications that exploit the ability of thermoelectric generators to operate at low source temperatures [16,17]. In such applications, the generator is likely to be made from alloys of bismuth telluride [18], though the dopant levels will be different from those in materials for refrigeration modules $[19,20]$.

In the bismuth telluride alloys there are some problems that arise when one attempts to extrapolate to the high temperature properties from low temperature data. For example, the data vary from one author to another depending on the processes that have been used in preparing the thermoelectric material. Also, even at low temperatures, the accurate determination of the thermoelectric properties is not easy.

Clearly, then, any extrapolation applies only for samples prepared by a particular technique. However, even though the absolute value of a particular property may be uncertain, the determination of the ratio of this parameter at different temperatures should be more precise.

It is generally thought that acoustic-mode lattice scattering is predominant in bismuth telluride and its alloys. If we assume that the carriers reside in a single parabolic band, the plot of Seebeck coefficient against $\log T$ in the classical extrinsic region should be linear with a slope equal to $3 k / 2 e$. Moreover, the carrier mobility in the same region should be proportional to $T^{-3 / 2}$. Neither of these features is strictly displayed by either n-type or p-type bismuth telluride. Nevertheless, the prediction that the electrical conductivity for a given Seebeck coefficient should be independent of temperature is quite accurate, as shown in Table 1. However, even for bismuth telluride, it is probably best to extrapolate from the observed temperature variation of mobility rather than the theoretical behaviour.

Table 1. Electrical conductivity of n-type and p-type $\mathrm{Bi}_{2} \mathrm{Te}_{3}$ at different temperatures for a given Seebeck coefficient, chosen as $-170 \mu \mathrm{V} / \mathrm{K}$ for n-type material and $160 \mu \mathrm{V} / \mathrm{K}$ for p-type material.

\begin{tabular}{cccc}
\hline Type & $\sigma$ at $150 \mathrm{~K}$ & $\sigma$ at 300 K & Ratio of conductivities, $\sigma_{\mathbf{3 0 0}} / \sigma_{150}$ \\
\hline $\mathrm{n}$ & $1.5 \times 10^{5} \mathrm{ohm}^{-1} \mathrm{~m}^{-1}$ & $1.65 \times 10^{5} \mathrm{ohm}^{-1} \mathrm{~m}^{-1}$ & 1.10 \\
$\mathrm{p}$ & $1.55 \times 10^{5} \mathrm{ohm}^{-1} \mathrm{~m}^{-1}$ & $1.4 \times 10^{5} \mathrm{ohm}^{-1} \mathrm{~m}^{-1}$ & 0.90 \\
\hline
\end{tabular}

Unlike many other thermoelectric materials, bismuth telluride is anisotropic, with both the carrier mobility and the lattice conductivity being dependent on the direction of transport [21]. Because the ratio of the hole mobility in the $c$ direction to the $a$ direction happens to be almost the same (about one-half) as the ratio of the lattice conductivities, the figure of merit of p-type material is almost independent of direction. However, the mobility of electrons in the $c$ direction is no more than about onequarter of that along the $a$ axis so that the highest figure of merit for n-type bismuth telluride is only reached for aligned material. Single crystals are not needed provided that the grains are all aligned with the $a$ axes parallel to a certain direction. The degree of crystallographic alignment must be taken into account in predicting the high temperature properties.

A feature of bismuth telluride that is particularly relevant when it is used in power generation is the rather small energy gap [22,23]. At $300 \mathrm{~K}$ the gap is no more than about $5 k T$ in width so that, even at this temperature, minority carrier effects must be taken into account when optimising the doping level 
and, thus, the Seebeck coefficient. As the temperature rises, the effect of the minority carriers on both the Seebeck coefficient and the electronic thermal conductivity becomes increasingly important $[24,25]$.

Figures 2 and 3 show the variation of $Z T$ with temperature for selected p-type and n-type materials, respectively. In all the examples, $Z T$ rises with temperature at first and then falls off as bipolar effects make themselves felt. Both the rise at low temperatures and the falling off at higher temperatures are consistent with our theoretical expectations. It should be noted that the lower curve in Figure 3 represents early observations (published over 40 years ago), but it is included since a wider range of temperature is covered than that in most reports.

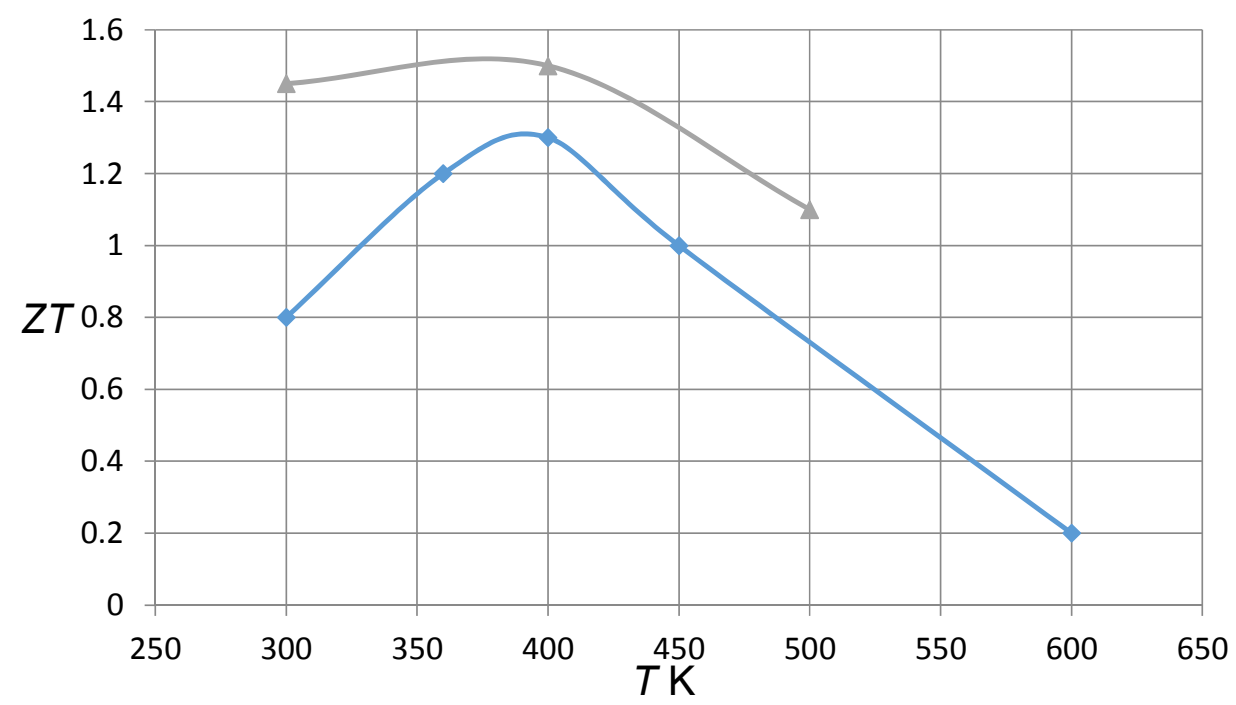

Figure 2. Plot of ZT against $\mathrm{T}$ for selected p-type bismuth telluride alloys. The upper curve shows the observations of Xie et al. [26] on nanocomposites made by melt spinning and spark-plasma sintering. The lower curve shows the data of $\mathrm{Li}$ et al. [27] on bismuth-antimony telluride prepared by mechanical alloying and spark plasma sintering.

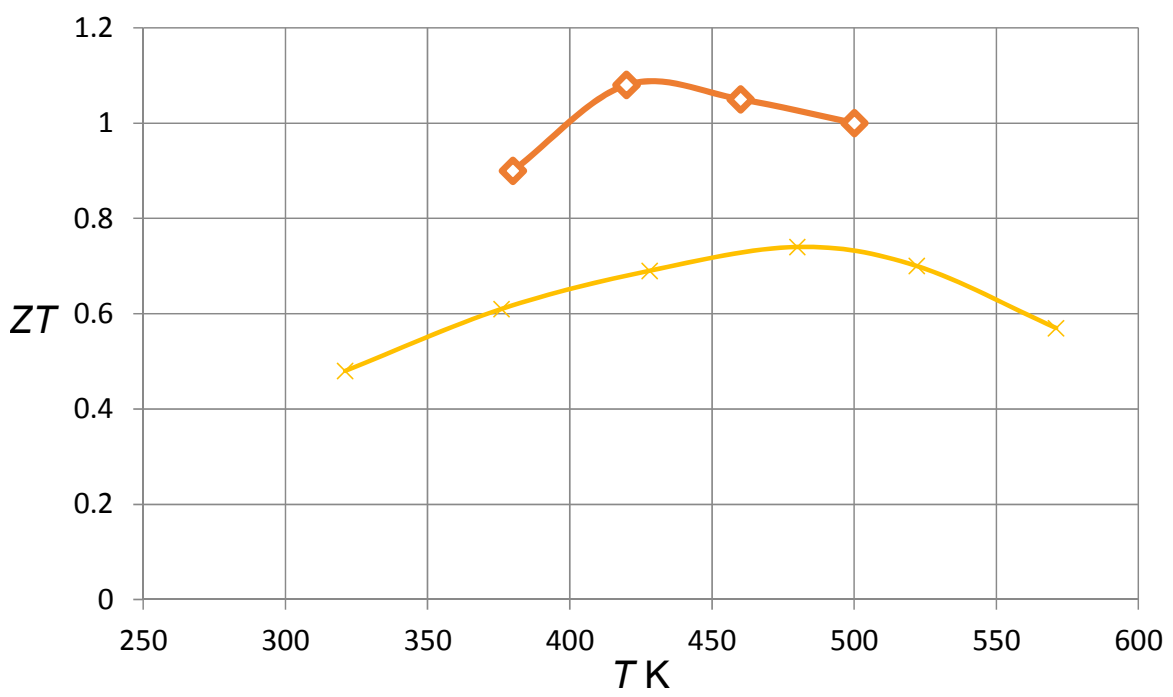

Figure 3. Plot of ZT against $\mathrm{T}$ for selected n-type bismuth telluride alloys. The upper curve shows the observations of $\mathrm{Wu}$ et al. [28]. The lower curve shows the early data of Imamuddin and Dupre [29] on bismuth seleno-telluride. 
Both figures make it clear that appreciably higher values of $Z T$ would be obtained at higher temperatures if minority carrier conduction could be suppressed to some extent by increasing the energy gap.

It has long been established that the energy gap increases as the selenium content in bismuth seleno-telluride alloys is increased [30], as shown in Figure 4, and, for these alloys, the lattice conductivity is lower than that of bismuth telluride itself. Nevertheless, the selenium content in the n-type bismuth seleno-telluride alloys used in refrigeration modules rarely exceeds $10 \%$ because of a reduction in the electron mobility at higher concentrations. However, it is likely that bismuth telluride alloys for generation should contain an increased level of selenium. Hopefully, the larger energy gap might compensate for any fall in the mobility.

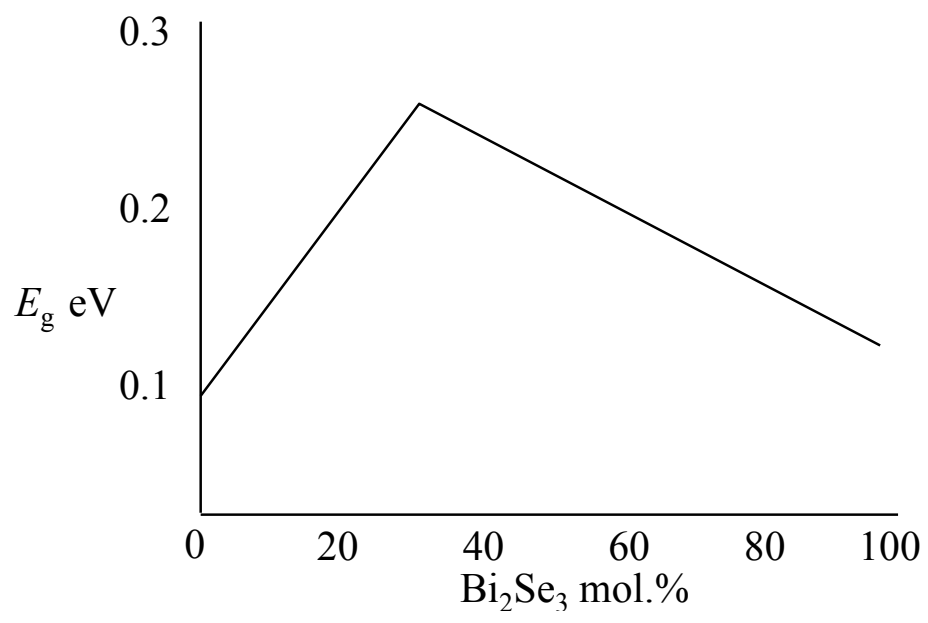

Figure 4. Schematic plot of energy gap against molar percentage of bismuth selenide in bismuth seleno-telluride alloys.

The p-type bismuth telluride alloys in refrigeration modules are normally based on the composition $\mathrm{Bi}_{0.5} \mathrm{Sb}_{1.5} \mathrm{Te}_{3}$. For many years it was thought that the energy gap in $\mathrm{Sb}_{2} \mathrm{Te}_{3}$ was smaller than that of $\mathrm{Bi}_{2} \mathrm{Te}_{3}$, since it is difficult to obtain a Seebeck coefficient as high as $200 \mu \mathrm{V} / \mathrm{K}$ in this compound. However, recent work suggests that this is a problem arising from considerable lack of stoichiometry; it is difficult to achieve doping that will compensate for an excess of antimony. Recently a Seebeck coefficient of $200 \mu \mathrm{V} / \mathrm{K}$ at $500 \mathrm{~K}$ has been observed by Sun et al. [31] indicating that the energy gap of $\mathrm{Sb}_{2} \mathrm{Te}_{3}$ may actually be larger than that of $\mathrm{Bi}_{2} \mathrm{Te}_{3}$. It may, therefore, be possible to improve the value of $Z T$ in bismuth telluride alloys at high temperatures by increasing both the selenium and antimony concentrations. Be that as it may, it has been claimed that $Z T$ exceeding unity can be achieved in bismuth telluride alloys up to a temperature of at least $500 \mathrm{~K}$.

It might also be possible to increase the Seebeck coefficient at high temperatures by introducing ionized impurity scattering since this would enlarge the average energy transported by the charge carriers [32]. However, it remains to be demonstrated that this effect can bring any overall benefit.

It is interesting to look at the relative magnitudes of the electronic and lattice contributions to the thermal conductivity in the bismuth telluride alloy system. We consider material that has a Seebeck coefficient of $2.5 \mathrm{k} / \mathrm{e}$, that is $216 \mu \mathrm{V} / \mathrm{K}$, a value that must be close to the optimum. It is then found that the electronic component is about one-half of the lattice conductivity. The lattice conductivity is influenced 
by both phonon-phonon scattering and alloy scattering. As a reasonable approximation, we can suppose that the lattice thermal resistivity can be calculated as the sum of the resistivities due to both forms of scattering. Thus, the total thermal conductivity is made up partly of an electronic contribution that rises with temperature, at the optimised Seebeck coefficient, and a lattice component, the temperature dependence of which lies between $T^{-1}$ and $T^{0}$. It is then not unreasonable to assume that the total thermal conductivity is more or less independent of temperature. Since the optimised power factor is also approximately independent of temperature, we expect $Z T$ to be proportional to $T$ for optimised material. For $Z T$ plots of single samples, as in Figures 2 and 3, we can expect $Z T$ to increase faster than $T$ up to the point at which the optimum Seebeck coefficient is reached.

\section{Application to Silicon-Germanium}

$\mathrm{Si}-\mathrm{Ge}$ is a thermoelectric generator material that is used at moderately high temperatures. Basu et al. [33] reported a very large value of $Z T$ for $\mathrm{Si}_{80} \mathrm{Ge}_{20}$ that was prepared by a hot-press sintering technique. There is the suggestion that the material is nanostructured. One of the features of this work is the claim that $Z T$ is equal to 1.84 at $1073 \mathrm{~K}$. The results of measurements on the thermoelectric parameters from room temperature up to about $1100 \mathrm{~K}$ are given. We have attempted to fit the data of Basu et al. to our calculations of the figure of merit at high temperatures, from the observed properties at low temperatures. The properties were found to vary according to the ball-milling time and we use the results for the (best) sample made from powders milled for $72 \mathrm{~h}$.

According to Basu et al., the material displaying $Z T=1.84$ at high temperatures had a $Z T$ value of 0.2 at $300 \mathrm{~K}$ with a Seebeck coefficient of $-1.68 \mathrm{k} / \mathrm{e}$. This implies a value of the parameter $\beta$ for $\mathrm{n}$-type material at this temperature of 0.06 . The review of Si-Ge by Vining [34] gives the energy gap of $0.99 \mathrm{eV}$ and the solidus temperature (which presumably sets an upper limit to the operating range) is $1550 \mathrm{~K}$. The parameter $c$, equal to $\beta_{\mathrm{p}} / \beta_{\mathrm{n}}$, will have little influence but a value of 0.5 is probably appropriate for alloys of the composition selected.

The only question that remains, then, is the variation of $\beta_{\mathrm{n}}$ with temperature. Since the alloy has a much lower lattice thermal conductivity than either Si or Ge we might expect $\lambda_{\mathrm{L}}$ to be more or less independent of temperature. In this case, $\beta_{n}$ would be proportional to $T$ if acoustic mode lattice scattering of the electrons were the only mechanism. However, this would lead to a value for $Z T$ at $1100 \mathrm{~K}$ of only 0.59. The observations of Basu et al. fit much better to the assumption that $\beta_{\mathrm{n}}$ is proportional to $T^{2}$ or that there is an even stronger temperature-dependence. In Figure 5, we show the variation of $Z T$ with temperature from Basu's data and from our calculations. 


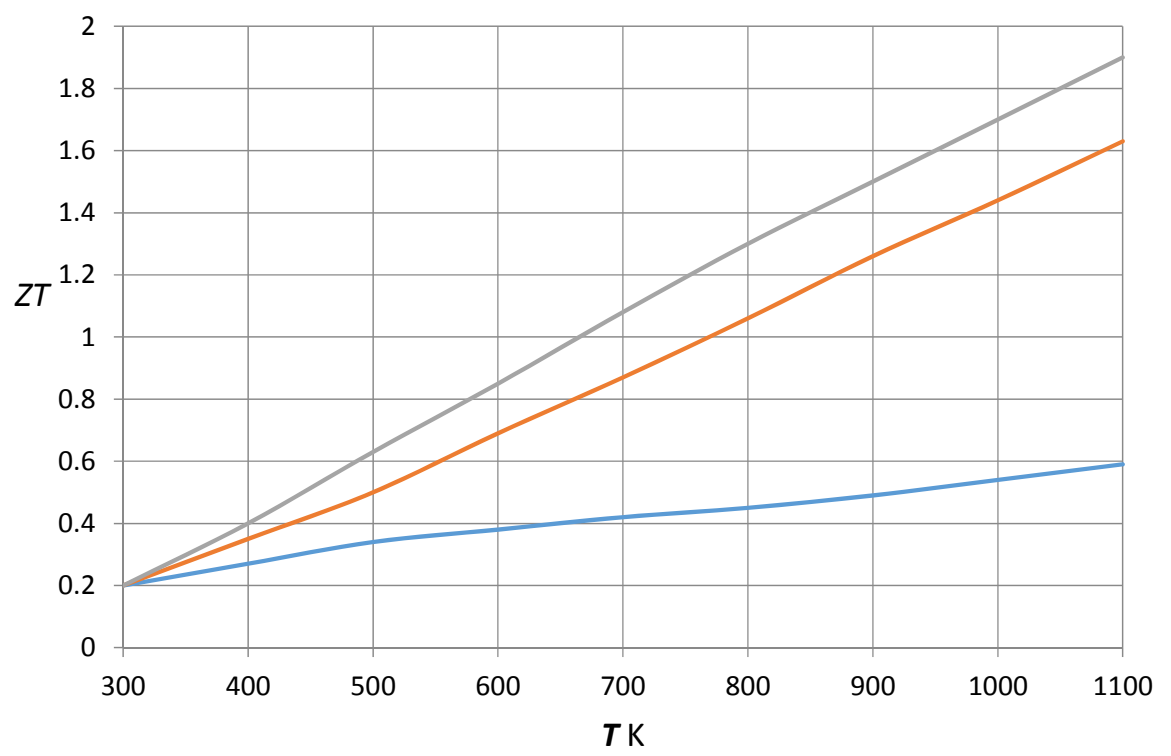

Figure 5. Plot of $\mathrm{ZT}$ against temperature for $\mathrm{Si}_{80} \mathrm{Ge}_{20}$. The top curve is from the results of Basu et al. [33]. The bottom curve is a calculation based on the assumption that $\beta_{\mathrm{n}}$ is proportional to $\mathrm{T}$ and the middle curve is for $\beta_{\mathrm{n}}$ proportional to $\mathrm{T}^{2}$.

It is noted that similar though somewhat lower $Z T$ results than those of Basu et al. have been reported previously by Bathula et al. [35] and Wang et al. [36]. The values of $Z T$ at $900{ }^{\circ} \mathrm{C}(1173 \mathrm{~K})$ given by these authors are 1.45 and 1.3 respectively. From the data of these authors, it also appears that $\beta_{\mathrm{n}}$ is approximately proportional to $T^{2}$.

We can see how the variation of $\beta_{n}$ with temperature arises when we look at the observations of Bathula et al. The lattice conductivity is not quite independent of temperature as it changes from $1.8 \mathrm{~W} / \mathrm{m} \mathrm{K}$ at $300 \mathrm{~K}$ to $1.6 \mathrm{~W} / \mathrm{m} \mathrm{K}$ at $600 \mathrm{~K}$. However, the main factor that controls the temperature dependence of $\beta_{\mathrm{n}}$ is the quantity $\sigma_{0}$, that is $2 e \mu\left(2 \pi m * k T / h^{2}\right)^{3 / 2}$. For acoustic-mode lattice scattering the carrier mobility should be proportional to $T^{-3 / 2}$, which would mean a reduction by a factor of 2.8 over the temperature range 300 to $600 \mathrm{~K}$. The results of Bathula et al. show that the actual factor is only 1.4. Presumably, the relatively slow change of mobility with temperature and the significant change of lattice conductivity combine to yield the close to $T^{2}$ dependence for $\beta_{\mathrm{n}}$.

This study of Si-Ge indicates that the dependence of $\beta_{\mathrm{n}}$ on temperature is a parameter that needs to be known in predicting $Z T$ at high temperatures.

Incidentally, if the data provided by Basu et al. are reliable it is reasonable to expect that $Z T$ will rise above 2 at temperatures above $1200 \mathrm{~K}$ for suitably processed Si-Ge.

\section{Application to Magnesium-silicon-tin}

One of the potential thermoelectric generator materials is $\mathrm{Mg}_{2} \mathrm{Si}_{0.7} \mathrm{Sn}_{0.3}$. This alloy is featured in a review article by Zaitsev et al. [37]. The thermoelectric properties have been measured in the temperature range up to $850 \mathrm{~K}$. The energy gap of $0.65 \mathrm{eV}$ is not really small enough for bipolar effects to be observed at these temperatures but we need to take into account the fact that the lattice thermal resistivity has comparable contributions from alloy scattering and phonon-phonon scattering. We have set $c=1$ but the value of this parameter does not affect the results to any significant extent. 
The particular solid solution in which we are interested has a $Z T$ value of 0.85 at $800 \mathrm{~K}$. The Seebeck coefficient of the sample for which comprehensive data are presented is $-195 \mu \mathrm{V} / \mathrm{K}$, which is $-2.76 \mathrm{k} / \mathrm{e}$. At $400 \mathrm{~K}$ the same sample has a Seebeck coefficient of $-110 \mu \mathrm{V} / \mathrm{K}$, which is $-1.29 \mathrm{k} / \mathrm{e}$, and $Z T$ equal to 0.25 . This allows us to set $\beta_{\mathrm{n}}$ equal to 0.11 at $400 \mathrm{~K}$.

The paper by Zaitsev et al. gives the lattice thermal resistivity for a whole range of solid solutions at $300 \mathrm{~K}$. As one would expect, the thermal resistivity is lowest for the pure compounds $\mathrm{Mg}_{2} \mathrm{Si}$ and $\mathrm{Mg}_{2} \mathrm{Sn}$ and is highest for solid solutions with comparable concentrations of the two components. If we assume that the thermal resistivity due to phonon-scattering is that for the pure compound, the remaining resistivity is due to alloy scattering. The phonon-phonon component should be proportional to temperature while the alloy scattering component should be independent of temperature. The observations on $\mathrm{Mg}_{2} \mathrm{Si}_{0.7} \mathrm{Sn}_{0.3}$ are fitted by a lattice thermal resistivity equal to $(0.14 T / 300+0.36) \mathrm{m} \mathrm{K} / \mathrm{W}$.

We have calculated the behaviour of $Z T$ at elevated temperatures from the value at $400 \mathrm{~K}$ using 3 different assumptions:

(1) $\beta_{\mathrm{n}}$ proportional to $T$ (what one would expect if the lattice conductivity were independent of $T$ ).

(2) $\beta_{\text {n }}$ proportional to $T^{2}$ (to be expected if the lattice conductivity varied as $1 / T$ ).

(3) $\beta_{\mathrm{n}}$ proportional to $T(0.14 T / 300+0.36)$.

Assumption (3) should hold for the two different phonon-scattering mechanisms and for the quantity $\sigma_{0}$ being independent of temperature.

Figure 6 shows $Z T$ plotted against $T$ for the 3 different assumptions together with the observed behaviour as given in the paper by Zaitsev et al. The variation of $Z T$ with $T$ for Assumption (3) is very close to the experimental results. We can, therefore, predict $Z T$ for optimized samples up to the melting temperature with some confidence using Assumption (3). This has been done in Figure 7 up to $1050 \mathrm{~K}$. We do not know the solidus temperature for $\mathrm{Mg}_{2} \mathrm{Si}_{0.7} \mathrm{Sn}_{0.3}$, but it is likely to be higher than the melting point of $\mathrm{Mg}_{2} \mathrm{Sn}, 1051 \mathrm{~K}$. It is noted that $Z T$ in Figure 7 increases linearly with temperature above $600 \mathrm{~K}$, since, over the whole range, bipolar effects can be avoided.

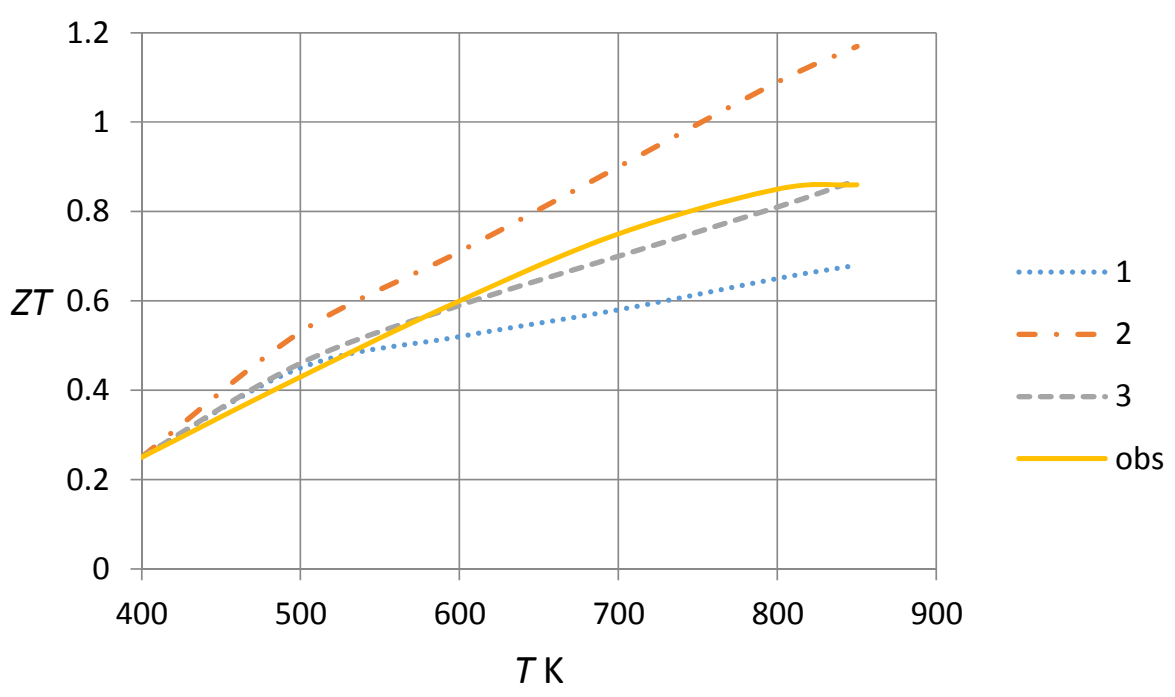

Figure 6. Plots of $\mathrm{ZT}$ against temperature for $\mathrm{Mg}_{2} \mathrm{Si}_{0.7} \mathrm{Sn} 0.3$. The plots 1,2 and 3 refer to the three different assumptions regarding the variation of $\beta$ with temperature. The fourth plot gives the data from Zaitsev et al. [37]. 


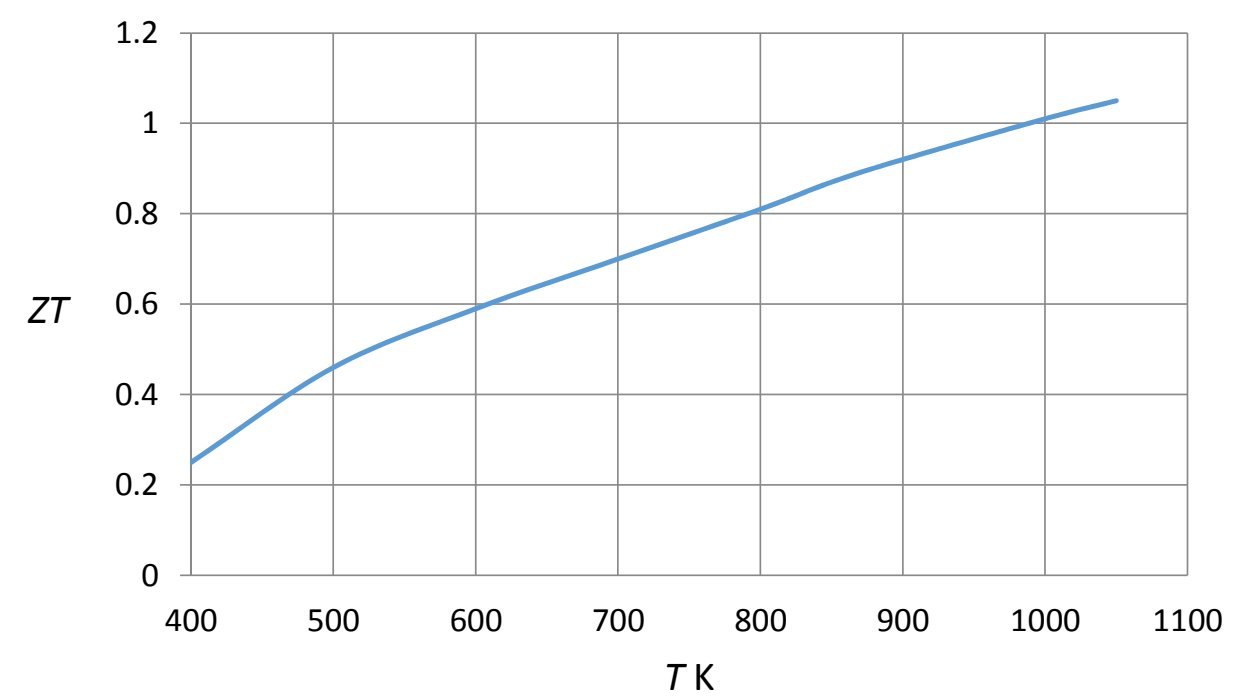

Figure 7. Plot of ZT against temperature for $\mathrm{Mg}_{2} \mathrm{Si}_{0.7} \mathrm{Sn}_{0.3}$ on the basis of assumption 3 .

\section{Application to Higher Manganese Silicide}

There do not seem to be many thermoelectric materials other than bismuth-antimony at low temperatures and bismuth telluride alloys, for which the energy gap is small enough to make minority carrier conduction a real problem, even at temperatures close to the melting point. However, one material that does seem to have a rather small gap is higher manganese silicide (HMS) [38]. The composition can range from $\mathrm{MnSi}_{1.70}$ to $\mathrm{MnSi}_{1.77}$. We shall look at complex-doped HMS which has an observed maximum $Z T$ of 0.7 for a Seebeck coefficient of about $210 \mu \mathrm{V} / \mathrm{K}$ at $800 \mathrm{~K}$. In fact, the decay of both $Z T$ and $\alpha$ above this temperature suggests that a higher $Z T$ might be achieved by suitable doping.

Figure 8 shows the parameters $\alpha, \sigma, \lambda$ and $Z T$ plotted against $T$ for complex-doped HMS over the range $300 \mathrm{~K}$ to $1000 \mathrm{~K}$. It is noted that the Seebeck coefficient reaches a maximum value of $210 \mu \mathrm{V} / \mathrm{K}$ at $800 \mathrm{~K}$. This allows us to estimate the energy gap to be $0.32 \mathrm{eV}$. We can use the observed values of $Z T$ in what is clearly the one-carrier conduction range to calculate $\beta_{p}$ for the majority carriers (holes) and its variation with temperature.

We find that the variation of $Z T$ and Seebeck coefficient in the temperature range $300 \mathrm{~K}$ to $700 \mathrm{~K}$ can be fitted by allowing $\beta_{\mathrm{p}}$ to vary as $T^{1.75}$. Inspection of Figure 8 suggests that the lattice conductivity does not fall with rise of temperature for this material, and the observed variation of $\beta_{\mathrm{p}}$ is associated primarily with the mobility of the carriers. According to Figure 8, $\sigma$ falls from $750 \Omega^{-1} \cdot \mathrm{cm}^{-1}$ to $560 \Omega^{-1} \cdot \mathrm{cm}^{-1}$ as the temperature changes from $300 \mathrm{~K}$ to $700 \mathrm{~K}$. This implies a mobility variation as $T^{-0.34}$. Such a mobility variation is very different from the $T^{-1.5}$ dependence that is expected for acoustic-mode lattice scattering. It seems that in many materials the reliable prediction of $Z T$ at high temperatures requires a determination of the temperature variation of $\beta_{\mathrm{n}}$ or $\beta_{\mathrm{p}}$.

We can use the observed $T^{1.75}$ dependence of $\beta_{\mathrm{p}}$ together with the energy gap of $0.32 \mathrm{eV}$ to predict the maximum value of $Z T$ up to $1000 \mathrm{~K}$. We have set the parameter $c$ equal to 1 for lack of knowledge of the properties of n-type HMS. The results of this calculation are shown in Figure 9. 


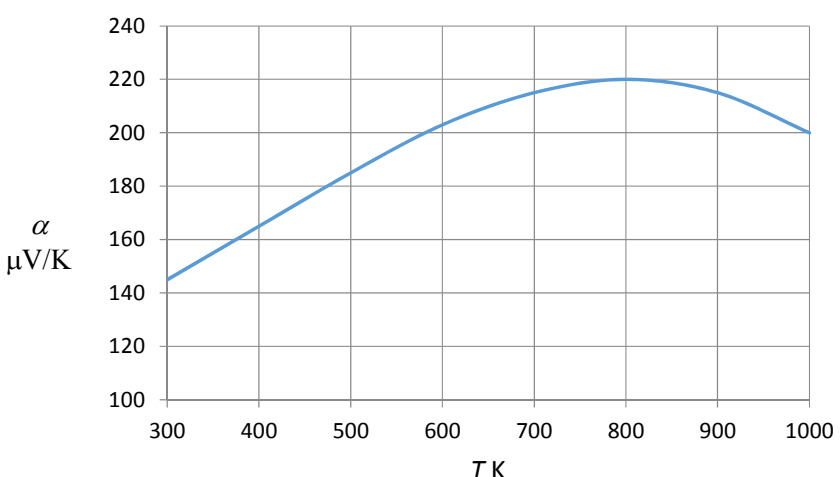

(a)

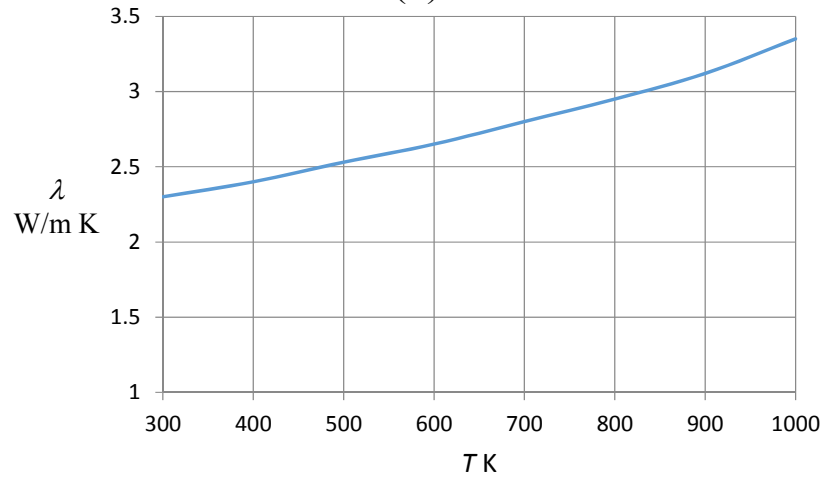

(c)

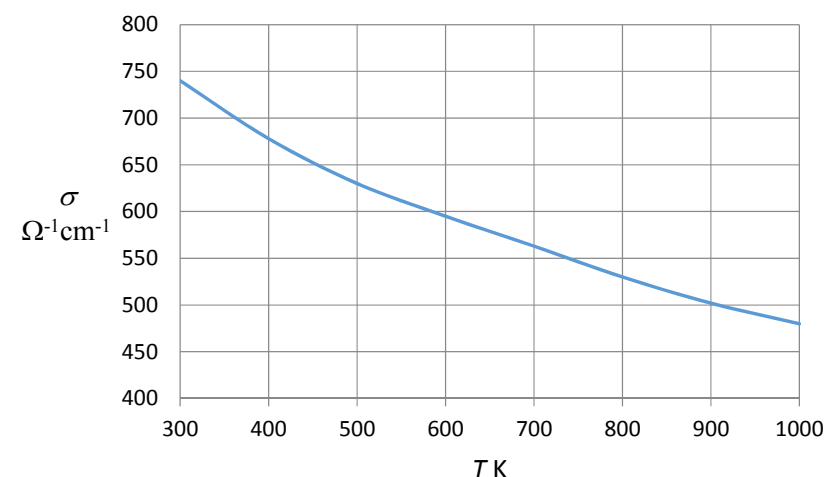

(b)

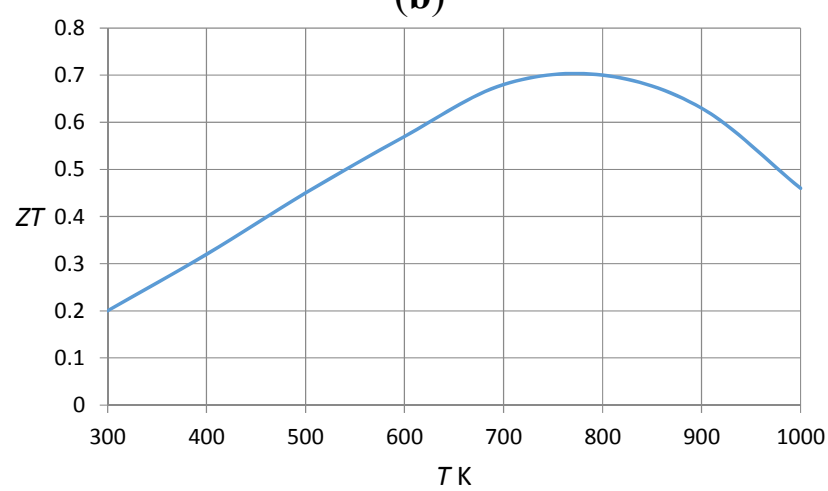

(d)

Figure 8. The thermoelectric properties of complex-doped HMS [38]. (a) Seebeck coefficient; (b) electrical conductivity; (c) thermal conductivity; and (d) ZT.

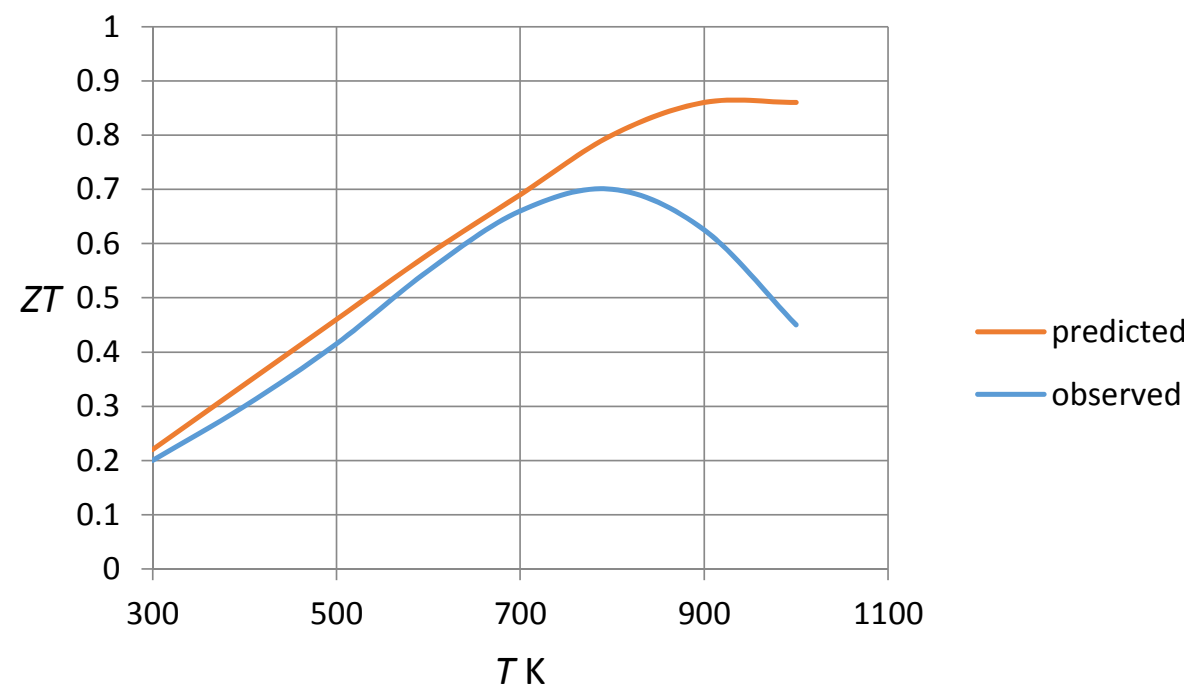

Figure 9. ZT plotted against temperature for complex-doped HMS. The upper curve is the maximum value predicted from the variation of $\beta_{\mathrm{p}}$ with temperature together with the energy gap as estimated from the maximum Seebeck coefficient. The lower curve represents the observations of Fedorov and Zaitsev [38].

Below $800 \mathrm{~K}$ there is little difference between the predictions and the observed behaviour (the small difference is due to the fact that the Seebeck coefficient has been optimised for the upper curve). The substantial difference above $800 \mathrm{~K}$ is due to the influence of minority carriers in reducing $Z T$ for the 
experimental sample. It is noted that a continuous rise of $Z T$ up to a temperature of $1000 \mathrm{~K}$ should be achievable if the effect of the minority carriers can be reduced by the addition of more acceptors.

\section{Conclusions}

Our objective has been the development of a simple method of predicting the $Z T$ at high temperatures for a material in which the energy gap is small enough for bipolar effects to be significant. We have found, in fact, that the problem is not quite as simple as we had hoped. This is because the temperature dependence of the parameter $\beta$ is not easily predicted. Thus, rather than using the data ane particular base temperature, we really need to know something about the temperature-dependence of $\sigma_{0}$ and $\lambda_{\mathrm{L}}$ before we can make a reliable estimate of the high-temperature properties. It should be stressed that the changes of $Z T$ with increasing temperature, as predicted in this paper, relate to optimised material rather than to samples with a given carrier concentration.

We have previously applied the techniques that are described here successfully to bismuth telluride and its alloys [39]. The energy gap in these materials is small enough for minority carrier conduction to require consideration even at room temperature.

\section{Conflicts of Interest}

The authors declare no conflicts of interest.

\section{References}

1. Goldsmid, H.J. Introduction to Thermoelectricity; Springer: Heidelberg, Germany, 2010.

2. Ioffe, A.F. Semiconductor Thermoelements and Thermoelectric Cooling; Infosearch: London, UK, 1957.

3. Vedernikov, M.V.; Kuznetsov, V.L. Cooling thermoelements with superconducting leg. In $C R C$ Handbook of Thermoelectrics; Rowe, D.M., Ed.; CRC Press: Boca Raton, FL, USA, 1995; p. 609.

4. Goldsmid, H.J.; Gopinathan, K.K.; Matthews, D.N.; Taylor, K.N.R.; Baird, C.A. High-Tc superconductors as passive thermo-elements. J. Phys. D 1988, 21, 344-348.

5. Rosi, F.D.; Abeles, B.; Jensen, R.V. Materials for thermoelectric refrigeration. J. Phys. Chem. Solids 1959, 10, 191-200.

6. Snyder, G.J. Thermoelectric power generation: Efficiency and compatibility. In Thermoelectrics Handbook: Macro to Nano; Rowe, D.M., Ed.; CRC Taylor and Francis, Boca Raton, FL, USA, 2006; pp. 9.1-9.26.

7. Martin, J.; Wong-Ng, W.; Green, M.L. Seebeck coefficient metrology: Do contemporary protocols measure up? J. Electron. Mater. 2015, 44, 1998-2006.

8. Wang, H.; Bai, S.; Chen, L.; Cuenat, A.; Joshi, G.; Kleinke, H.; König, J.; Lee, H.W.; Martin, J.; Oh, M.W.; et al. International round-robin study on thermoelectric transport properties of n-type half-Heusler from $300 \mathrm{~K}$ to 773 K. J. Electron. Mater. 2015, in press.

9. Chasmar, R.P.; Stratton, R. The thermoelectric figure of merit and its relation to thermoelectric generators. J. Electron. Control 1959, 7, 52-72. 
10. McDougall, J.; Stoner, E.C. The computation of Fermi-Dirac functions. Philos. Trans. A 1938, 237, 67-104.

11. Rhodes, P. Fermi-Dirac functions of integral order. Proc. R. Soc. A 1950, 204, 396-405.

12. Goldsmid, H.J.; Sharp, J.W. Estimation of the thermal band gap of a semiconductor from Seebeck measurements. J. Electron. Mater. 1999, 28, 869-872.

13. Muller, E.; Heiliger, W.; Reinshaus, P.; Sussman, H. Determination of the thermal band gap from the change of the Seebeck coefficient at the pn-transition in $\mathrm{Bi}_{0.5} \mathrm{Sb}_{1.5} \mathrm{Te}_{3}$. In Proceedings of the 15th International Conference on Thermoelectrics, Pasadena, CA, USA, 26-29 March 1996; IEEE: Piscataway, NJ, USA, 1996; p. 412.

14. Gibbs, Z.M.; Kim, H.S.; Wang, H.; Snyder, G.J. Band gap estimation from temperature dependent Seebeck measurement-Deviations from the $2 \mathrm{eS}_{\max } \mathrm{T}_{\max }$ relation. Appl. Phys. Lett. 2015, 106, 022112-1-022112-5.

15. Berger, L.I. General properties of semiconductors. In CRC Handbook of Chemistry and Physics, 91st ed.; CRC Press: Boca Raton, FL, USA, 2010-2011.

16. Min, G.; Rowe, D.M. Peltier devices as generators. In CRC Handbook of Thermoelectrics; Rowe, D.M., Ed.; CRC Press: Boca Raton, FL, USA, 1995; p. 479.

17. Matsuura, K.; Rowe, D.M. Low-temperature heat conversion. In CRC Handbook of Thermoelectrics; Rowe, D.M., Ed.; CRC Press: Boca Raton, FL, USA, 1995; p. 573.

18. Dashevsky, Z.; Drabkin, I.; Korotaev, V.; Rabinovich, D. Improved materials for thermoelectric conversion (generation). In Proceedings of the 16th International Conference on Thermoelectrics, Dresden, Germany, 26-29 August 1997; IEEE: Piscataway, NJ, USA, 1997; p. 382.

19. Kutasov, V.A.; Lukyanova, L.N.; Vedernikov, M.V. Shifting the maximum figure of merit of $(\mathrm{Bi}, \mathrm{Sb})_{2}(\mathrm{Te}, \mathrm{Se})_{3}$ thermoelectrics to lower temperatures. In Thermoelectrics Handbook: Macro to Nano; Rowe, D.M., Ed.; CRC Taylor and Francis: Boca Raton, FL, USA, 2006.

20. Kuznetsov, V.L. Functionally graded materials for thermoelectric applications. In Thermoelectrics Handbook: Macro to Nano; Rowe, D.M., Ed.; CRC Taylor and Francis: Boca Raton, FL, USA, 2012.

21. Delves, R.T.; Bowley, A.E.; Hazelden, D.W.; Goldsmid, H.J. Anisotropy of the electrical conductivity in bismuth telluride. Proc. Phys. Soc. 1961, 78, 838-844.

22. Austin, I.G. The optical properties of bismuth telluride. Proc. Phys. Soc. 1958, 72, 545-552.

23. Veis, A.N.; Luk'yanova, L.N.; Kutasov, V.A. Band gap and type of optical transitions at the interband absorption edge in solid solutions based on bismuth telluride. Phys. Solid State 2012, $54,2182-2188$.

24. Goldsmid, H.J. The electrical conductivity and thermoelectric power of bismuth telluride. Proc. Phys. Soc. 1958, 71, 633-646.

25. Goldsmid, H.J. The thermal conductivity of bismuth telluride. Proc. Phys. Soc. B 1956, 69, 203-209.

26. Xie, W.; Wang, S.; Zhu, S.; Ho, J.; Tang, X.; Zhang, Q.; Tritt, T.M. High performance Bi2 $\mathrm{Te}_{3}$ nanocomposites prepared by single-element-melt-spinning spark-plasma sintering. J. Mater. Sci. 2013, 48, 2745-2760.

27. Li, H.; Jing, H.; Han, Y.; Xu, Y.; Lu, G.Q.; Xu, L. Microstructure and transport properties of copper-doped p-type (BiSbTe) alloy prepared by mechanical alloying and subsequent spark plasma sintering. J. Alloy. Compd. 2013, 576, 369-374. 
28. Wu, F.; Song, H.; Gao, F.; Shi, W.; Jia, J.; Hu, X. Effects of different morphologies of Bi2 $\mathrm{Te}_{3}$ nanopowders on thermoelectric properties. J. Electron. Mater. 2013, 42, 1140-1145.

29. Imamuddin, M.; Dupre, A. Thermoelectric properties of p-type $\mathrm{Bi}_{2} \mathrm{Te}_{3}-\mathrm{Sb}_{2} \mathrm{Te}_{3}-\mathrm{Sb}_{2} \mathrm{Se}_{3}$ alloys and n-type $\mathrm{Bi}_{2} \mathrm{Te}_{3}-\mathrm{Bi}_{2} \mathrm{Se}_{3}$ alloys in the temperature range 300 to 600 K. Phys. Status Solidi A 1972, 10 , 415-424.

30. Greenaway, D.L.; Harbeke, G. Band structure of bismuth telluride, bismuth selenide and their respective alloys. J. Phys. Chem. Solids 1965, 26, 1585-1604.

31. Sun, S.; Peng, J.; Jin, R.; Song, S.; Zhu, P.; Xing, Y. Template-free solvothermal synthesis and enhanced thermoelectric performance of $\mathrm{Sb}_{2} \mathrm{Te}_{3}$ nanosheets. J. Alloy. Compd. 2013, 558, 6-10.

32. Goldsmid, H.J. Effect of the scattering law on the maximum Seebeck coefficient. J. Thermoelectr. 2006, 2, 5-8.

33. Basu, R.; Bhattacharya, S.; Bhatt, R.; Roy, M.; Ahmad, S.; Singh, A.; Navaneethan, M.; Hayakawa, Y.; Aswal, D.K.; Gupta, S.K. Improved thermoelectric performance of hot pressed nanostructured n-type Si-Ge bulk alloys. J. Mater. Chem. A 2014, 2, 6922-6930.

34. Vining, C. Silicon germanium. In CRC Handbook of Thermoelectrics; Rowe, D.M., Ed.; CRC Press: Boca Raton, FL, USA, 1994; p. 329.

35. Bathula, S.; Jayasimhadri, M.; Singh, N.; Srivastava, A.K.; Pulikkotil, J.; Dhar, A.; Budhani, R.C. Enhanced thermoelectric figure-of-merit in spark plasma sintered nanostructured n-type SiGe alloys. Appl. Phys. Lett. 2012, 101, 213902-1-213902-5.

36. Wang, X.W.; Lee, H.; Lan, Y.C.; Zhu, G.H.; Joshi, G.; Wang, D.Z.; Yang, J.; Muto, A.J.; Tang, M.Y.; Klatsky, J. et al. Enhanced thermoelectric figure of merit in nanostructured $n$-type silicon germanium bulk alloy. Appl. Phys. Lett. 2008, 93, 193121-1-193121-3.

37. Zaitsev, V.K.; Fedorov, M.I.; Eremin, I.S.; Gurieva, E.A. Thermoelectrics on the Base of Solid Solutions of $\mathrm{Mg}_{2} \mathrm{~B}^{\mathrm{IV}}$ Compounds ( $\left.\mathrm{B}^{\mathrm{IV}}=\mathrm{Si}, \mathrm{Ge}, \mathrm{Sn}\right)$. In Thermoelectrics Handbook, Macro to Nano; Rowe, D.M., Ed.; CRC Press: Boca Raton, FL, USA, 2006; doi:10.1201/9781420038903.ch29.

38. Fedorov, M.I.; Zaitsev, V.K. Thermoelectrics of transition metal silicides. In Thermoelectrics Handbook Macro to Nano; Rowe, D.M., Ed.; CRC Press: Boca Raton, FL, USA, 2006; pp. 31.1-31.19.

39. Goldsmid, H.J. Bismuth telluride and its alloys as materials for thermoelectric generation. Materials 2014, 7, 2577-2592.

(C) 2015 by the authors; licensee MDPI, Basel, Switzerland. This article is an open access article distributed under the terms and conditions of the Creative Commons Attribution license (http://creativecommons.org/licenses/by/4.0/). 